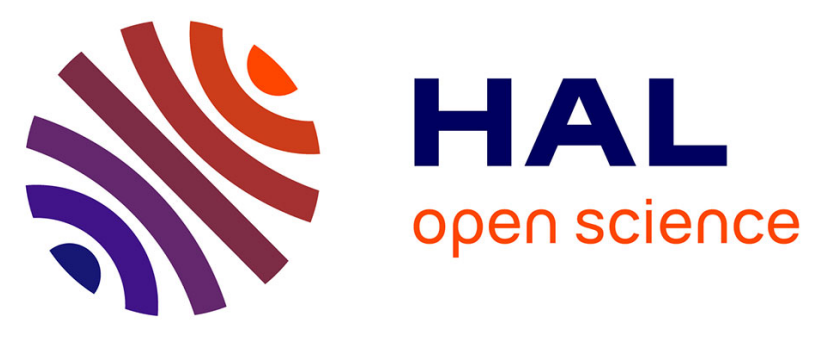

\title{
MIRU-VNTR allelic variability depends on Mycobacterium bovis clonal group identity
}

Amandine Hauer, Lorraine Michelet, Krystel de Cruz, Thierry Cochard, Maxime Branger, Claudine Karoui, Sylvie Henault, Franck Biet, María Laura Boschiroli

\section{To cite this version:}

Amandine Hauer, Lorraine Michelet, Krystel de Cruz, Thierry Cochard, Maxime Branger, et al.. MIRU-VNTR allelic variability depends on Mycobacterium bovis clonal group identity. Infection, Genetics and Evolution, 2016, 45, pp.165-169. 10.1016/j.meegid.2016.08.038 . hal-01595184

\author{
HAL Id: hal-01595184 \\ https://hal.science/hal-01595184
}

Submitted on 26 Sep 2017

HAL is a multi-disciplinary open access archive for the deposit and dissemination of scientific research documents, whether they are published or not. The documents may come from teaching and research institutions in France or abroad, or from public or private research centers.
L'archive ouverte pluridisciplinaire HAL, est destinée au dépôt et à la diffusion de documents scientifiques de niveau recherche, publiés ou non, émanant des établissements d'enseignement et de recherche français ou étrangers, des laboratoires publics ou privés. 


\title{
Short communication \\ MIRU-VNTR allelic variability depends on Mycobacterium bovis clonal group identity
}

\author{
Amandine Hauer ${ }^{\mathrm{a}, \mathrm{b}}$, Lorraine Michelet ${ }^{\mathrm{a}}$, Krystel De Cruz ${ }^{\mathrm{a}}$, Thierry Cochard ${ }^{\mathrm{b}}$, Maxime Branger ${ }^{\mathrm{b}}$, \\ Claudine Karoui a , Sylvie Henault ${ }^{a}$, Franck Biet ${ }^{\text {b,1 }}{ }^{\text {, María Laura Boschiroli }}{ }^{\text {a, }, 1}$ \\ a Université Paris-Est, Laboratoire National de Référence Tuberculose, Unité Zoonoses Bactériennes, Laboratoire de Santé Animale, ANSES, 94706 Maisons-Alfort Cedex, France \\ b INRA, Université de Tours, UMR1282, Infectiologie et Santé Publique, F-37380 Nouzilly, France
}

\section{A R T I C L E I N F O}

\section{Article history:}

Received 22 April 2016

Received in revised form 30 August 2016

Accepted 31 August 2016

Available online 01 September 2016

\begin{abstract}
A B S T R A C T
The description of the population of M. bovis strains circulating in France from 1978 to 2013 has highlighted the discriminating power of the MLVA among predominant spoligotype groups. In the present study we aimed to characterize clonal groups via MLVA and to better understand the strain's population structure. MLVA was performed with eight MIRU-VNTR loci, most of them defined by the Venomyc European consortium. The discriminatory index of each MLVA loci was calculated for SB0120, SB0134, SB0121 and the "F4-family", the main spoligotype groups in France. Differences in global DI per spoligotype, but also by locus within each spoligotype, were observed, which strongly suggest the clonal complex nature of these major groups. These MLVA results were compared to those of other European countries where strain collections had been characterized (Spain, Portugal, Italy, Northern Ireland and Belgium). Overall, QUB 3232 and ETR D are respectively the most and the least discriminative loci, regardless of the strains geographical origin. However, marked DI differences are observed in the rest of the MIRU-VNTR loci, again highlighting that strain genetic variability in a country depends on the dominant existing clonal complexes. A web application for M. bovis, including spoligotyping and MIRUVNTR typing data, was developed to allow inter-laboratory comparison of field isolates.

In conclusion, combination of typing methods is required for $M$. bovis optimum discrimination and differentiation of groups of strains. Thus, the loci employed for MLVA in a country should be those which are the most discriminative for the clonal complexes which characterize their M. bovis population.
\end{abstract}

(c) 2016 Published by Elsevier B.V.

\section{Introduction}

France is an officially bovine tuberculosis (bTB) free EU member still presenting several outbreaks of the disease every year. These outbreaks are concentrated in few regions where wildlife is also affected by the disease. To better understand the epidemiology of the disease and the reasons of this persistence as well as defining the phylogeny of the strains, spoligotyping and multi-locus variable number tandem repeat analysis (MLVA) were employed in a large and long-established $M$. bovis strain collection. These two methods are largely used for conventional genotyping and have become indispensable to study the molecular epidemiology of bTB worldwide (Gormley et al., 2014). This study was performed on 2332 strains isolated from animal outbreaks during

\footnotetext{
* Corresponding author.

E-mail address: maria-laura.boschiroli@anses.fr (M.L. Boschiroli).

${ }^{1}$ Franck Biet and María Laura Boschiroli contributed equally to this work.
}

a period of 35 years (1978-2013) widely representing the French epidemiological bTB situation (Hauer et al., 2015). MLVA revealed a very high genetic variability among the four main spoligotype SB0120, SB0121, SB0134 and "F4" family composed of strains belonging to 25 spoligotypes that lack spacer 33 and present a truncated repeat in QUB 26 (Hauer et al., 2015).

To determine whether the use of MIRU-VNTR, in addition to subtyping strains, could be indicative of clonal group identity, we analyzed the allelic diversity of each of the 6 loci proposed by the Venomyc European consortium plus loci ETR C and QUB 26 on our large field isolates' set. Our results support that spoligotyping and MLVA are useful not only for molecular epidemiology, but are also phylogenetically informative, predicting diverse strain lineages at a national level for this Mycobacterium tuberculosis complex (MTBC) species. This study also provides a new web application dedicated to the genotyping of $M$. bovis including spoligotyping and MIRU-VNTR typing for the scientific community and veterinary health services. 


\section{Materials and methods}

\subsection{Bacterial strains}

This study was performed on 2332 field $M$. bovis isolates belonging to the French bTB National Reference Laboratory (Anses) collection, where they are currently maintained and stored. They were isolated between 1978 and 2013 from 1751 cattle, 86 wild boar, 56 badgers, 28 goats, 21 domestic and exotic animals such as monkeys (baboon (Papio sp.)), chimpanzee (Pan sp.), macaque (Macaca sp.) and felids (puma (Puma concolor) and tiger (Panthera tigris)), 11 red deer, 11 pigs, 8 wildlife animals and 3 sheep. $M$. bovis isolates were identified by spoligotyping as described below. For livestock breakdowns, a herd-based criterium was used for strain choice: at least 1 strain/outbreak was genotyped by both genotyping methods (spoligotyping and MLVA). If there was more than one spoligotype profile in the same herd during one year, at least one strain of each spoligotype profile was genotyped by MLVA. The totality of wildlife, zoo or other domestic animal isolates were fully genotyped by both methods.

\subsection{Spoligotyping}

Spoligotyping was performed as described by (Zhang et al., 2010), using TB-SPOL kits purchased from Beamedex® (Beamedex ${ }^{\circledR}$ SAS, Orsay, France) on BioPLex200/Luminex 200® as described by Hauer et al. (2015). Spoligotypes have been named according to an agreed international convention (www.mbovis.org).

\subsection{MLVA typing using MIRU and VNTR markers}

Multi-Locus Variable numbers tandem repeats Analysis (MLVA) profile identification (Frothingham and Meeker-O'Connell, 1998; Roring et al., 2002) was performed by Genoscreen, Lille, France, using PCR amplification targeting genetic loci including mycobacterial MIRU-VNTR. Analysis was based on 8 loci, ETR A (VNTR2165), ETR B (VNTR2461), ETR C (VNTR577), ETR D (MIRU4 or VNTR580), QUB 11a (VNTR2163a), QUB 11b (VNTR2163b), QUB 26 (VNTR4052) and QUB 3232 (VNTR3232), chosen in the framework of an European consortium on their degree of polymorphism and their ability to discriminate local strains (Supply et al., 2006).

\subsection{Discriminatory index calculation}

The discriminatory index (DI) allows to measure variations in specific genetic regions used in the employed genotyping methods. DI of spoligotyping and MLVA was determined using the Hunter and Gaston formula DI $=1-\left(1 \div \mathrm{N}(\mathrm{N}-1) \sum_{j=1}^{s} \mathrm{nj}(\mathrm{n} j-1)\right)$ where $N$ is the total number of strains in the typing scheme, $s$ is the total number of distinct patterns discriminated by each typing method and strategy, and $n j$ is the number of strains belonging to the $j$ th pattern (Hunter and Gaston, 1988). The DI of the combination of spoligotyping and MLVA was calculated on a dendrogram (Categorical values) distance represented by UPGMA (Unweighted Pair Group Method with Aritmetic mean) clustering method for MLVA) built by Bionumerics 7.0 software (Applied Maths, St-Martin-Latem, Belgium) (data not shown). DI and confidence intervals at 95\% of each MIRU-VNTR locus were performed with the VNTR-Diversity and Confidence Extractor (V-DICE) website (http:// www.hpa-bioinformatics.org).

\subsection{M. bovis genotyping database}

A freely accessible web application called "M.bovis Datatype" including MLVA and spoligotyping profiles was developed (http://mbov-type. tours.inra.fr). This database gathers all profiles analyzed in this study which are those previously published by Hauer et al. (Hauer et al.,
2015). The database information includes incrementing MIRU-VNTR and spoligotype profiles with corresponding alleles/repeat numbers at each locus, and an application to automatically obtains octal code. This database allows generating octal codes defined by the CDC (complementary to hexacodes on Mbovis.org). Through this application, interlaboratory comparisons of MLVA and spoligotyping profiles can be performed with those of existing referenced strains.

\section{Results and discussion}

Spoligotyping of the $2332 \mathrm{M}$. bovis strains investigated in this study identified 153 different profiles (Table S1). But as previously described (Hauer et al., 2015), 69\% of the strains are grouped in only ten spoligotypes of which three are dominant: SB0120, SB0134 and SB0121 (30, 12 and 6\% respectively) (Table S1). The spoligotyping method is very useful for strain differentiation, with an estimated DI of 0.882 , but does not allow tracing up studies when spoligotypes are dominant and widespread (Skuce et al., 2002).

To improve the discrimination of strains within the main spoligotypes and improve epidemiological studies, MLVA with a set of eight loci (ETR A, ETR B, ETR C, ETR D, QUB 11a, QUB 11b, QUB 26, QUB 3232), was used (Table 1 and Figs. 1 and 2). On the 2332 strains examined, 471 different MLVA profiles have been identified of which 256 ( $11 \%$ of the strains included in this study) were obtained only once during the 1978-2011 period. MLVA was thus able to sub-discriminate the three main spoligotypes (Table 1). As shown in previous studies, each MLVA loci seem more or less suitable to discriminate $M$. bovis strains depending on the spoligotype group (Gormley et al., 2014). With this unique collection of $M$. bovis strains, regarding its size and diversity, the DI of each MIRU-VNTR locus was calculated for all strains and for strains within each major spoligotype group as shown in Fig. 1 and Fig. 2. Collectively, these findings established that ETR A is the most discriminating locus ( $\mathrm{DI}=0.712$ ), followed in descending order by QUB 3232 (DI = 0.695), ETR B (DI =0.691), QUB 26 (DI = 0.687), QUB $11 \mathrm{a}(\mathrm{DI}=0.678), \mathrm{QUB} 11 \mathrm{~b}(\mathrm{DI}=0.614), \mathrm{ETR} C(\mathrm{DI}=0.360)$ and ETR $\mathrm{D}$ which is the most stable and less discriminative $(\mathrm{DI}=0.163)$ of them. DI of MLVA was also calculated for SB0120, SB0134 and SB0121 and the "F4-family", the largest spoligotype groups, for which clear DI differences could be noticed (Figs. 1 and 2 and Table 2A). SB0120, the most prevalent spoligotype in France (30\% of strains), is also highly diverse (DI: 0.858). However SB0121, which represents $6.2 \%$ of strains, presents the highest DI (0.931) while SB0134, accounting for $12 \%$ of strains, shows a lower DI (0.677). In Italy SB0120 is also the principal spoligotype and, as in France, ETR B discloses the higher discriminatory power (0.68 and 0.65 respectively) (Table 2A). The results on SB0121 strains are consistent with those previously reported from the Iberian Peninsula (Spain and Portugal) where this spoligotype is the most frequent and for which MLVA DI was up to 0.98 (Duarte et al., 2010; Rodriguez-Campos et al., 2013) (Table 2B). As suggested by Rodriguez-Campos et al., the high diversity of these strains, which belong to the European 2 complex could be explained either by a higher mutation rate or by the long lasting presence of the pathogen in

Table 1

Distribution of field isolates of $M$. bovis by genotyping and MLVA spoligotype.

\begin{tabular}{|c|c|c|c|c|}
\hline Family $^{\mathrm{a}}$ & $\begin{array}{l}\text { No. of } \\
\text { spoligotypes }\end{array}$ & $\begin{array}{l}\text { No. of MLVA } \\
\text { profiles }\end{array}$ & $\begin{array}{l}\text { No. of } \\
\text { isolates }\end{array}$ & $\begin{array}{l}\% \text { of } \\
\text { strains }\end{array}$ \\
\hline SB0120 & 1 & 154 & 768 & 33 \\
\hline SB0134 & 1 & 53 & 272 & 12 \\
\hline SB0121 & 1 & 50 & 157 & 7 \\
\hline F4-family & 27 & 111 & 552 & 24 \\
\hline Sub-total & 30 & 368 & 1749 & 76 \\
\hline Other spoligotypes ${ }^{b}$ & 123 & 103 & 583 & 24 \\
\hline Total & 153 & 471 & 2332 & 100 \\
\hline
\end{tabular}

a Spoligotypes predominately present in France and used in this study.

b Previously described (Hauer et al., 2015). 


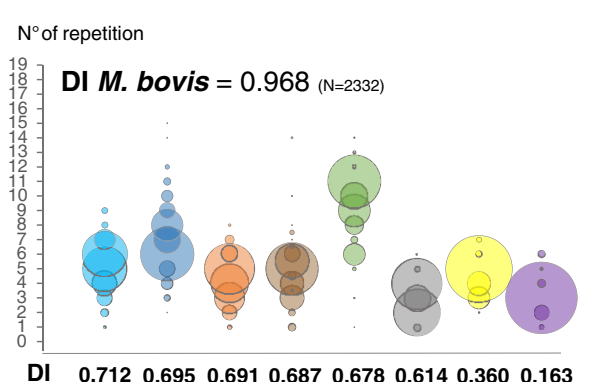

DI $\quad 0.712 \quad 0.695 \quad 0.6910 .687 \quad 0.678 \quad 0.614 \quad 0.3600 .163$

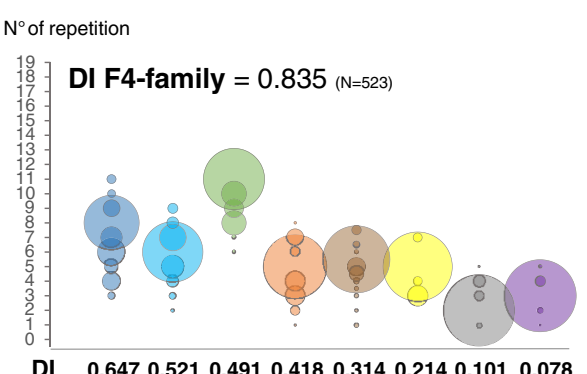

DI $\quad 0.6470 .521 \quad 0.4910 .418 \quad 0.314 \quad 0.214 \quad 0.101 \quad 0.078$

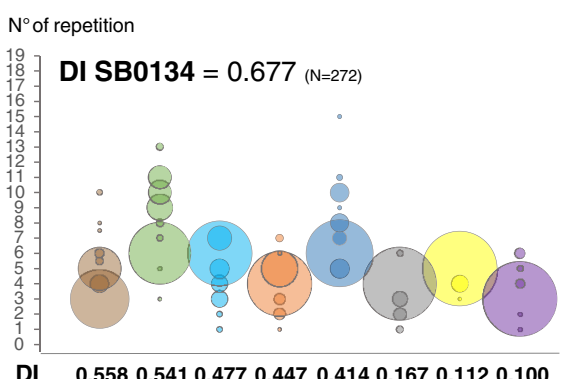

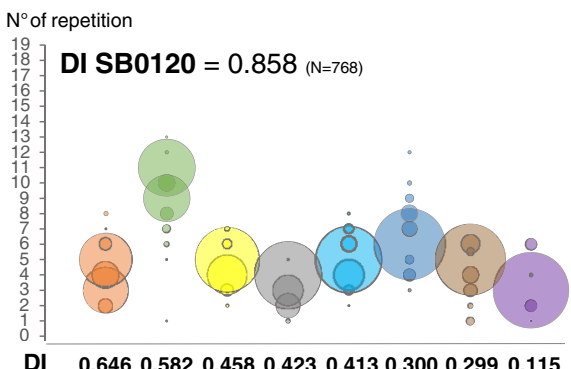

DI $\quad 0.6460 .5820 .4580 .423 \quad 0.4130 .3000 .2990 .115$

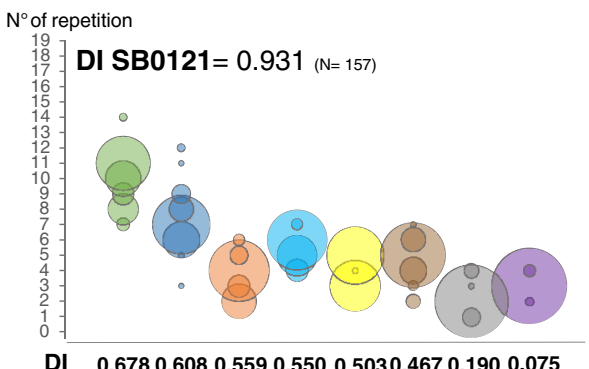

DI 0.6780 .6080 .5590 .5500 .5030 .4670 .1900 .075

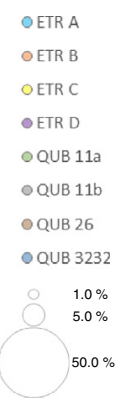

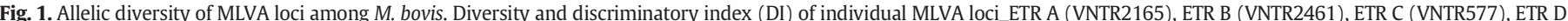

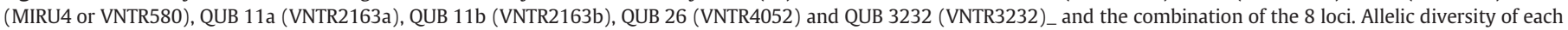

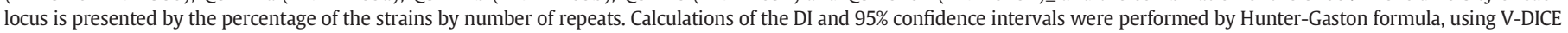
website on 2332 isolates for M. bovis, 768 isolates for SB0120, 272 isolates for SB0134, 157 isolates for SB0121 and 523 isolates for the F4-family.

mainland Europe, which gave more opportunity for evolution (Rodriguez-Campos et al., 2012). On the contrary, the lower diversity for SB0134 could be explained by the more recent emergence and expansion of these strains in France in the last 10 years (Hauer et al., 2015). As for the "F4 family" that accounts for $22 \%$ of strains in this collection, the DI of MLVA, 0.835 , is quite high. This family exclusively found in France, mainly in the South, is composed of strains belonging to 25 spoligotypes that lack spacer 33 and present a truncated repeat in QUB 26 (Haddad et al., 2001; Hauer et al., 2015). In Belgium the most common spoligotype is SB0162, which is, by contrast, rare in
France. For this spoligotype, together with ETR D, the least discriminative locus is ETR C (DI = 0.18) (Table 2B) (Allix et al., 2006). In Northern Ireland, where the majority of strains belong to the European 1 complex, discrimination of these strains is maximal with QUB 3232, while QUB 11b is the least variable (Skuce et al., 2005).

New and interesting information provided by this thorough study based on the DI per MIRU-VNTR locus is the differential genetic variability highlighted among these families suggesting a possible use in phylogenetic studies. Maximal discrimination power is observed in completely different loci per spoligotype: ETR B for SB0120, QUB 26

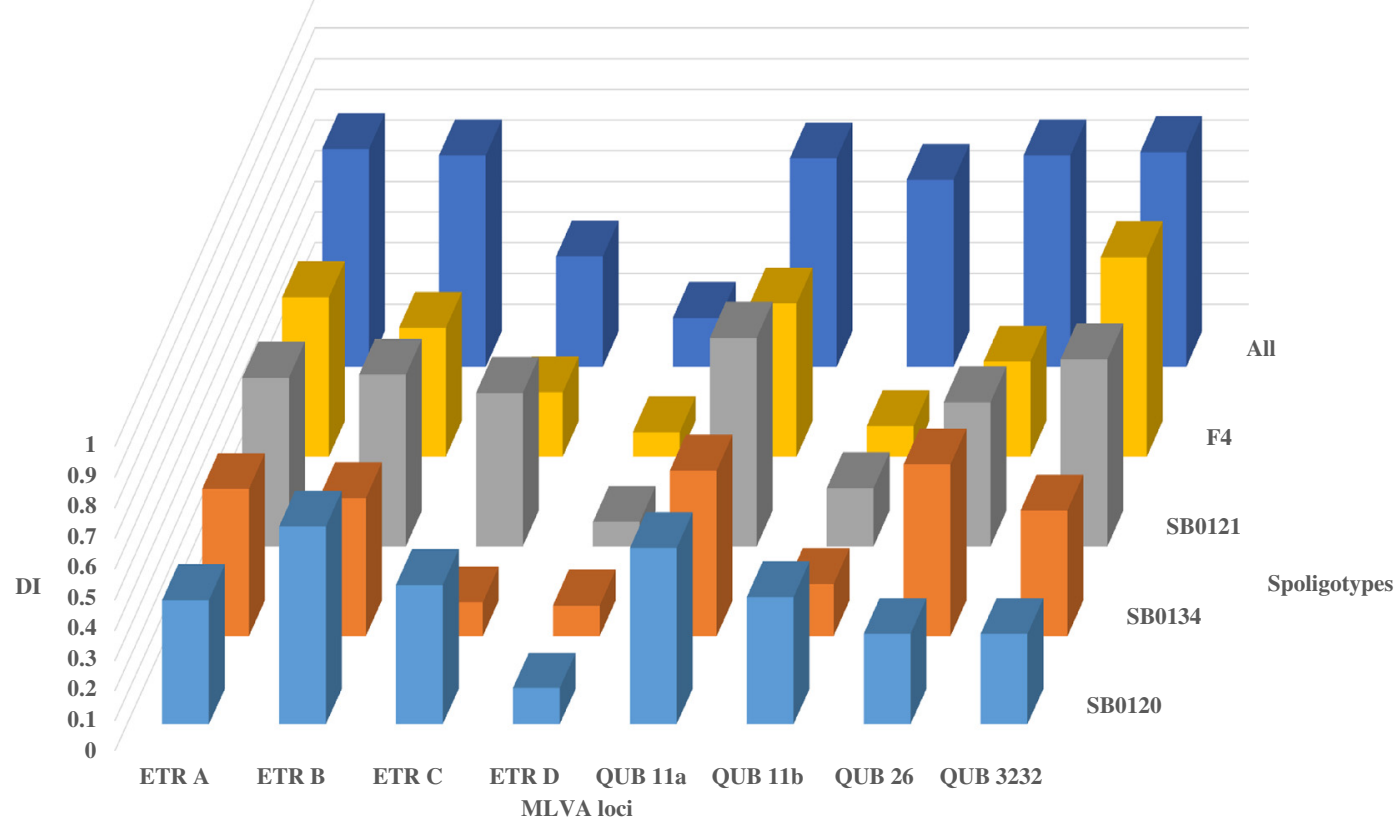

Fig. 2. Comparison of discriminatory index (DI) of individual MLVA loci by spoligotype family. 
Table 2

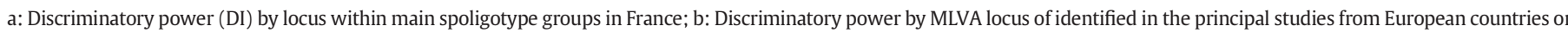
regions.

\begin{tabular}{|c|c|c|c|c|c|c|c|c|c|c|}
\hline \multirow{2}{*}{ Country } & \multirow{2}{*}{ No. of strains } & \multirow{2}{*}{ Spoligotypes } & \multicolumn{8}{|c|}{ DI by locus } \\
\hline & & & ETR A & ETR B & ETR C & ETR D & QUB 11a & QUB 11b & QUB 26 & QUB 3232 \\
\hline \multirow{5}{*}{ France } & 2332 & All (100\%) & 0.71 & 0.70 & 0.36 & 0.16 & 0.68 & 0.61 & 0.69 & 0.69 \\
\hline & 768 & SB0120 (33\%) & 0.41 & 0.65 & 0.46 & 0.12 & 0.58 & 0.42 & 0.30 & 0.30 \\
\hline & 272 & SB0134 (12\%) & 0.48 & 0.45 & 0.11 & 0.10 & 0.54 & 0.17 & 0.56 & 0.41 \\
\hline & 157 & SB0121 (7\%) & 0.55 & 0.56 & 0.50 & 0.08 & 0.68 & 0.19 & 0.47 & 0.61 \\
\hline & 523 & F4 (22\%) & 0.52 & 0.42 & 0.21 & 0.08 & 0.50 & 0.10 & 0.31 & 0.65 \\
\hline
\end{tabular}

B. Comparison of allelic polymorphism by MLVA locus determined from different geographical regions

\begin{tabular}{|c|c|c|c|c|c|c|c|c|c|c|}
\hline \multirow{2}{*}{ Country } & \multirow{2}{*}{ No. of strains } & \multirow{2}{*}{ Major spoligotypes } & \multicolumn{8}{|c|}{ DI by locus } \\
\hline & & & ETR A & ETR B & ETR C & ETR D & QUB 11a & QUB 11b & QUB 26 & QUB 3232 \\
\hline France $^{a}$ & 2332 & All & 0.71 & 0.70 & 0.36 & 0.16 & 0.68 & 0.61 & 0.69 & 0.69 \\
\hline Belgium $^{\mathrm{b}}$ & 82 & SB0162 & 0.66 & 0.69 & 0.18 & 0.18 & - & - & - & - \\
\hline Iberian Peninsula $^{c}$ & 115 & SB0121 & 0.64 & 0.53 & - & 0.20 & 0.44 & 0.08 & 0.35 & 0.82 \\
\hline Italy $^{\mathrm{d}}$ & 747 & SB0120 & 0.57 & 0.68 & 0.21 & 0.22 & - & - & - & - \\
\hline Portugal $^{\mathrm{e}}$ & 141 & SB0121 & 0.62 & 0.63 & 0.46 & 0.33 & 0.65 & 0.60 & - & 0.80 \\
\hline Northern Ireland ${ }^{\mathrm{f}}$ & 461 & SB0140 & 0.39 & 0.30 & - & - & 0.44 & 0.14 & 0.30 & 0.53 \\
\hline
\end{tabular}

a This study.

b Allix et al. (2006).

c Rodriguez-Campos et al. (2013).

d Boniotti et al. (2009).

e Duarte et al. (2010)

f Skuce et al. (2005).

for SB0134, QUB 11a for SB0121 and QUB 3232 for F4-family. ETR D is the least discriminating locus on all the populations (SB0120, SB0121, SB0134 and the F4-family). This genetic trait, i.e. differences in discrimination by locus in each family, supports the definition of main clonal complexes in France as suggested before (Hauer et al., 2015). Thus, the choice of the most appropriate set of loci for a finest MLVA typing should take in consideration the DI by locus of the most important spoligotypes and their derived spoligotypes (rare or orphan types) which constitute the population of M. bovis strains in a country.

The comparison with the reports of other countries that present similar or different spoligotype groups is also variable depending on the dominant strains in the countries (Table 2B) (Gormley et al., 2014). However, a common trait is that globally ETR D is poorly discriminative for $M$. bovis in contrast to the higher discrimination values observed for this locus in Mycobacterium tuberculosis (Supply et al., 2006) or Mycobacterium caprae (Prodinger et al., 2005) ( 0.55 and 0.58 respectively). In general, QUB 3232 seems to be very useful regardless of the strain set (Table $2 \mathrm{~A}$ and $\mathrm{B}$ ).

Studies conducted to determine a reference set of MLVA markers for $M$. bovis are fewer than studies on M. tuberculosis. However, in Europe, a network of laboratories has agreed on a consensus of MLVA loci for the use with M. bovis (Supply et al., 2006). Thus MLVA typing has been used in many countries for epidemiological studies although methodology standardization between laboratories as well as archiving and sharing knowledge of existing and of newly defined $M$. bovis genotypes, including MIRU-VNTR and spoligotype profiles, need to be managed. Therefore a web application called "M.Bovis Datatype" was developed (http://mbov-type.tours.inra.fr). This freely accessible service allows users to compare spoligotypes and MIRU-VNTR profiles of their strains. This database gathers all profiles obtained in this study, of which some of them have also been described in the literature. The database information includes incrementing spoligotype and MLVA profiles with corresponding alleles/repeat numbers at each locus. All information can be exported under EXCEL or PDF format. An extensive documentation regarding the genotyping method, protocols, and primer sequences is provided to the users.

In conclusion, this study, conducted in an important and diverse collection of strains, illustrates that MLVA loci sets should be optimized using panels of MIRU-VNTR suited for the clonal complexes composing the $M$. bovis population in a region or in a country where several identified widespread clonal complexes prevail. In addition a web application dedicated to the genotyping of $M$. bovis has been created to facilitate inter-laboratory comparison and exchange of genotyping and epidemiological data on M. bovis.

Supplementary data to this article can be found online at http://dx. doi.org/10.1016/j.meegid.2016.08.038.

\section{Acknowledgments}

This work was partly funded by a CNRS-MIE project. Amandine Hauer is an INRA-ANSES doctoral fellow. We are grateful to Victoria Boschiroli for her useful comments on the article.

\section{Reference}

Allix, C., Walravens, K., Saegerman, C., Godfroid, J., Supply, P., Fauville-Dufaux, M., 2006 Evaluation of the epidemiological relevance of variable-number tandem-repeat genotyping of Mycobacterium bovis and comparison of the method with IS6110 restriction fragment length polymorphism analysis and spoligotyping. J. Clin. Microbiol. 44, 1951-1962.

Boniotti, M.B., Goria, M., Loda, D., Garrone, A., Benedetto, A., Mondo, A., Tisato, E., Zanoni, M., Zoppi, S., Dondo, A., Tagliabue, S., Bonora, S., Zanardi, G., Pacciarini, M.L., 2009. Molecular typing of Mycobacterium bovis strains isolated in Italy from 2000 to 2006 and evaluation of variable-number tandem repeats for geographically optimized genotyping. J. Clin. Microbiol. 47, 636-644.

Duarte, E.L., Domingos, M., Amado, A., Cunha, M.V., Botelho, A., 2010. MIRU-VNTR typing adds discriminatory value to groups of Mycobacterium bovis and Mycobacterium caprae strains defined by spoligotyping. Vet. Microbiol. 143, 299-306.

Frothingham, R., Meeker-O'Connell, W.A., 1998. Genetic diversity in the Mycobacterium tuberculosis complex based on variable numbers of tandem DNA repeats. Microbiology 144 (Pt 5), 1189-1196.

Gormley, E., Corner, L.A., Costello, E., Rodriguez-Campos, S., 2014. Bacteriological diagnosis and molecular strain typing of Mycobacterium bovis and Mycobacterium caprae. Res. Vet. Sci. 97 (Suppl), S30-S43.

Haddad, N., Ostyn, A., Karoui, C., Masselot, M., Thorel, M.F., Hughes, S.L., Inwald, J., Hewinson, R.G., Durand, B., 2001. Spoligotype diversity of Mycobacterium bovis strains isolated in France from 1979 to 2000. J. Clin. Microbiol. 39, 3623-3632.

Hauer, A., De Cruz, K., Cochard, T., Godreuil, S., Karoui, C., Henault, S., Bulach, T., Banuls, A.L., Biet, F., Boschiroli, M.L., 2015. Genetic evolution of Mycobacterium bovis causing tuberculosis in livestock and wildlife in France since 1978. PLoS One 10, e0117103.

Hunter, P.R., Gaston, M.A., 1988. Numerical index of the discriminatory ability of typing systems: an application of Simpson's index of diversity. J. Clin. Microbiol. 26, 2465-2466.

Prodinger, W.M., Brandstatter, A., Naumann, L., Pacciarini, M., Kubica, T., Boschiroli, M.L., Aranaz, A., Nagy, G., Cvetnic, Z., Ocepek, M., Skrypnyk, A., Erler, W., Niemann, S., 
Pavlik, I., Moser, I., 2005. Characterization of Mycobacterium caprae isolates from Europe by mycobacterial interspersed repetitive unit genotyping. J. Clin. Microbiol. 43, 4984-4992.

Rodriguez-Campos, S., Schurch, A.C., Dale, J., Lohan, A.J., Cunha, M.V., Botelho, A., De Cruz K., Boschiroli, M.L., Boniotti, M.B., Pacciarini, M., Garcia-Pelayo, M.C., Romero, B., de Juan, L., Dominguez, L., Gordon, S.V., van Soolingen, D., Loftus, B., Berg, S., Hewinson, R.G., Aranaz, A., Smith, N.H., 2012. European 2-a clonal complex of Mycobacterium bovis dominant in the Iberian Peninsula. Infect. Genet. Evol. 12, 866-872.

Rodriguez-Campos, S., Navarro, Y., Romero, B., de Juan, L., Bezos, J., Mateos, A., Golby, P., Smith, N.H., Hewinson, G.R., Dominguez, L., Garcia-de-Viedma, D., Aranaz, A., 2013. Splitting of a prevalent Mycobacterium bovis spoligotype by variable-number tandem-repeat typing reveals high heterogeneity in an evolving clonal group. J. Clin. Microbiol. 51, 3658-3665.

Roring, S., Scott, A., Brittain, D., Walker, I., Hewinson, G., Neill, S., Skuce, R., 2002. Development of variable-number tandem repeat typing of Mycobacterium bovis: comparison of results with those obtained by using existing exact tandem repeats and spoligotyping. J. Clin. Microbiol. 40, 2126-2133.

Skuce, R., McCorry, T.P., McCarroll, J., Roring, S., Scott, A.E., Brittain, D., Hughes, M.S., Glyn Hewinson, R., Neill, S.D., 2002. Discrimination of Mycobacterium tuberculosis complex bacteria using novel VNTR-PCR targets. Microbiology 148, 519-528.
Skuce, R.A., McDowell, S.W., Mallon, T.R., Luke, B., Breadon, E.L., Lagan, P.L., McCormick, C.M., McBride, S.H., Pollock, J.M., 2005. Discrimination of isolates of Mycobacterium bovis in Northern Ireland on the basis of variable numbers of tandem repeats (VNTRs). Vet. Rec. 157, 501-504

Supply, P., Allix, C., Lesjean, S., Cardoso-Oelemann, M., Rusch-Gerdes, S., Willery, E., Savine, E., de Haas, P., van Deutekom, H., Roring, S., Bifani, P., Kurepina, N., Kreiswirth, B., Sola, C., Rastogi, N., Vatin, V., Gutierrez, M.C., Fauville, M., Niemann, S., Skuce, R., Kremer, K., Locht, C., van Soolingen, D., 2006. Proposal for standardization of optimized mycobacterial interspersed repetitive unit-variable-number tandem repeat typing of Mycobacterium tuberculosis. J. Clin. Microbiol. 44, 4498-4510.

Zhang, J., Abadia, E., Refregier, G., Tafaj, S., Boschiroli, M.L., Guillard, B., Andremont, A. Ruimy, R., Sola, C., 2010. Mycobacterium tuberculosis complex CRISPR genotyping: improving efficiency, throughput and discriminative power of 'spoligotyping' with new spacers and a microbead-based hybridization assay. J. Med. Microbiol. 59, 285-294. 